\title{
A New Numerical Approach for the Laminar Boundary Layer Flow and Heat Transfer along a Stretching Cylinder Embedded in a Porous Medium with Variable Thermal Conductivity
}

\author{
S. Shateyi ${ }^{1}$ and G. T. Marewo ${ }^{2}$ \\ ${ }^{1}$ Department of Mathematics, University of Venda, Private Bag X5050, Thohoyandou 0950, South Africa \\ ${ }^{2}$ Department of Mathematics, University of Swaziland, Private Bag 4, Kwaluseni M201, Switzerland \\ Correspondence should be addressed to S. Shateyi; stanford.shateyi@univen.ac.za
}

Received 16 September 2013; Revised 25 October 2013; Accepted 28 October 2013

Academic Editor: Morteza Rafei

Copyright (c) 2013 S. Shateyi and G. T. Marewo. This is an open access article distributed under the Creative Commons Attribution License, which permits unrestricted use, distribution, and reproduction in any medium, provided the original work is properly cited.

\begin{abstract}
The study presents an axisymmetric laminar boundary layer flow of a viscous incompressible fluid and heat transfer over a stretching cylinder embedded in a porous medium. A suitable similarity transformation is employed to transform the partial differential equations corresponding to the momentum and heat equations into nonlinear ordinary differential equations. The resultant ordinary differential equations are then solved using a successive relaxation method (SRM). The effects of significant parameters on the velocity and temperature profiles have been analyzed graphically. The obtained results are also compared with previously published results in some special cases and were found to be in excellent agreement. The skin friction as well as the heat transfer rate at the surface are increased as the values of the curvature parameter increase.
\end{abstract}

\section{Introduction}

The study of boundary layer flow and heat transfer due to stretching flat plates or cylinders has gained considerable attention due to its applications in fibre technology and extrusion processes, as well as theoretical interest. Such applications include the cooling of metallic plates, the boundary layer along a liquid film in condensation processes, boundary layer along material handling conveyers, among others. The rate of heat transfer at the stretching surfaces determines the quality of the final product. Sakiadis [1,2] pioneered the study of boundary layer flow on a moving continuous solid surface. Thereafter, Crane [3] extended this concept to a stretching sheet with linear surface speed. The study presented an exact solution for the steady two-dimensional flow over a stretching surface in a quiescent fluid. Since then, considerable work has been done by many authors who considered various aspects of this important field (e.g., Laha et al. [4]; Afzal [5]; Prasad et al. [6]; Abel and Mahesha [7]; Abel et al. [8]; Bataller [9]).
Flow over cylinders are of two types. They may be considered to be two-dimensional if the body radius is large compared to the boundary layer thickness. On the other hand, if the cylinder is thin/slender, the radius of the cylinder may be of the same order as that of the boundary layer thickness. In such a scenario, the flow may be considered as axisymmetric instead of two-dimensional (Elbarbary and Elgazery [10], Datta et al. [11], among others).

Lin and Shih [12,13] studied laminar boundary layer and heat transfer along horizontally and vertically moving cylinders with constant velocity. The studies found that similarity solutions could not be obtained due to the curvature effect of the cylinder. However, Ishak and Nazar [14] showed that the similarity solutions may be obtained by assuming that the cylinder is stretched with linear velocity in the axial direction and ascertained that their study may be regarded as the extension of the papers by Grubka and Bobba [15] and Ali [16] from a stretching sheet to a stretching cylinder. 
Mukhopadhyay [17] presented an axisymmetric laminar boundary layer mixed convection flow of a viscous incompressible fluid and heat transfer towards a stretching cylinder embedded in a porous medium. Mukhopadhyay [18] analyzed the flow and thermal characteristics of boundary layer axisymmetric flow and heat transfer along a stretching cylinder in a porous medium. Rangi and Ahmad [19] numerically studied the boundary layer flow of viscous incompressible fluid over a stretching cylinder with variable conductivity. Elbashbeshy et al. [20] discussed laminar boundary layer flow of an incompressible viscous fluid along a stretching horizontal surface embedded in a porous medium in the presence of a heat source or sink with suction/injection. Varjravelu et al. [21] presented numerical solutions to the unsteady convective boundary layer flow of a viscous fluid at a vertical stretching surface with variable transport properties and thermal radiation.

Sarkar et al. [22] investigated the buoyancy driven mixed convective flow and heat transfer characteristics of waterbased nanofluid past a cylinder. Soid et al. [23] studied a steady two-dimensional magnetohydrodynamics flow and heat transfer over a stretching vertical sheet influenced by radiation and porosity. Elbashbeshy et al. [24] discussed laminar boundary layer flow of an incompressible viscous fluid along a stretching horizontal cylinder in a porous medium. More recently, Chatterjee and Raja [25] investigated numerically the problem of fluid flow and heat transfer subjected to superimposed thermal buoyancy around tandem square cylinders. More recently, Mukhopadhyay and Gorla [26] presented an axisymmetric laminar boundary layer flow of a viscous incompressible fluid and heat transfer towards a stretching cylinder.

The present study seeks to extend the work of Rangi and Ahmad [19] to include porosity while also extending the work of Mukhopadhyay [18] to include variable thermal conductivity. The study will be carried out using a newly developed numerical scheme known as the successive relaxation method. This method is based on simple iteration schemes which are formed by reducing the order of the momentum equation which is then followed by linearizing the nonlinear equations. For more details, please see [27]. The rest of the paper is outlined as follows: in Section 2 we give the model formulation of the problem; in Section 3, the successive relaxation method is given in detail; in Section 4, the results and discussion are given; and Section 5 gives the conclusions based on the findings.

\section{Mathematical Formulation}

We consider the steady, axisymmetric boundary layer flow of a viscous and incompressible fluid along a continuously stretching horizontal cylinder of radius $R$ embedded in a porous medium. We assume that the stretching velocity $U(x)$ and the surface temperature are of the forms $U(x)=U_{0}(x / l)$ and $T_{w}(x)=T_{\infty}+T_{\infty}(x / l)$, respectively, where $U_{0}$ and $T_{0}$ are constants and $T_{\infty}$ and $l$ are the ambient temperature and characteristic length, respectively. Under these assumptions together with the boundary layer approximations, the equations which model the current problem under consideration are given as

$$
\begin{gathered}
\frac{\partial u}{\partial x}(r u)+\frac{\partial v}{\partial r}(r v)=0 \\
u \frac{\partial u}{\partial x}+v \frac{\partial u}{\partial r}=\frac{\nu}{r} \frac{\partial}{\partial r}\left(r \frac{\partial u}{\partial r}\right)-\frac{\nu}{K_{p}} u \\
u \frac{\partial T}{\partial x}+v \frac{\partial T}{\partial r}=\frac{1}{r} \frac{\partial}{\partial r}\left(\alpha r \frac{\partial T}{\partial r}\right),
\end{gathered}
$$

where $u$ and $v$ are velocity components in the $x$ - and $r$ directions, respectively, $T$ is the fluid temperature, $\alpha$ is the thermal diffusivity, $v$ is the kinematic viscosity, and $K_{p}$ is the permeability parameter. The appropriate boundary conditions are

$$
\begin{gathered}
u=U_{w}(x), \quad v=0, \quad T=T_{w}(x), \quad \text { at } r=R, \\
u \longrightarrow 0, \quad T \longrightarrow T_{\infty} \quad \text { as } r \longrightarrow \infty .
\end{gathered}
$$

2.1. Similarity Transformation. We then transform the momentum and energy equations into the corresponding ordinary differential equations using the following (Ishak and Nazar [14]):

$$
\begin{gathered}
\eta=\frac{r^{2}-R^{2}}{2 R}\left(\frac{U_{w}}{v x}\right)^{1 / 2}, \\
\psi(x, r)=R \sqrt{v x U_{w}} f(\eta), \quad \theta(\eta)=\frac{T-T_{\infty}}{T_{w}-T_{\infty}},
\end{gathered}
$$

where $f(\eta)$ is the dimensionless stream function, $\eta$ is the similarity variable, and $\theta$ is the dimensionless temperature. The continuity equation is automatically satisfied through the variables. For liquid metals, it had been observed that the thermal conductivity $\alpha$ varies with temperature in an approximately linear relationship in the range from $0^{\circ} \mathrm{F}$ to $400^{\circ} \mathrm{F}$. To that end, we therefore assume $\alpha=\alpha_{\infty}(1+\epsilon \theta)$. Upon introducing the relations (5) into (2)-(3), we obtain the following nonlinear system of ordinary differential equations:

$$
\begin{aligned}
& (1+2 \gamma \eta) f^{\prime \prime \prime}+2 \gamma f^{\prime \prime}+f f^{\prime \prime}-f^{\prime 2}-K f^{\prime}=0, \\
& (1+2 \gamma \eta) \theta^{\prime \prime}+2 \gamma \theta^{\prime}+\operatorname{Pr} f \theta^{\prime} \\
& \quad+\epsilon\left[(1+2 \gamma \eta)\left(\theta^{\prime 2}+\theta \theta^{\prime \prime}\right)+2 \gamma \theta \theta^{\prime}\right]=0,
\end{aligned}
$$

subject to the boundary conditions

$$
\begin{gathered}
f(0)=0, \quad f^{\prime}(0)=1, \quad \theta(0)=1, \\
f^{\prime} \longrightarrow 0, \quad \theta \longrightarrow 0 \quad \text { as } \eta \longrightarrow \infty,
\end{gathered}
$$

where prime denotes the differentiation with respect to $\eta, \gamma=$ $R^{-1}\left(\nu l / U_{0}\right)^{1 / 2}$ is the curvature parameter, $K=\nu l / K_{p} U_{0}$ is the permeability parameter, and $\operatorname{Pr}=\mu c_{p} / k$ is the Prandtl number.

The physical quantities of interest in engineering are the skin friction coefficient $C_{f}$ and the local Nusselt number $\mathrm{Nu}_{x}$ which are defined as

$$
C_{f}=\frac{2 \tau_{w}}{\rho U_{w}^{2}}, \quad \mathrm{Nu}_{x}=\frac{x q_{w}}{\kappa\left(T_{w}-T_{\infty}\right)},
$$


respectively, where $\tau_{w}=\mu(\partial u / \partial r)_{r=R}$ is the surface shear stress and $q_{w}=-\kappa(\partial T / \partial r)_{r=R}$ is the surface heat flux. Upon substituting the similarity transformations (5) into (10) we obtain

$$
\frac{1}{2} \sqrt{\mathrm{Re}_{x}}=f^{\prime \prime}(0), \quad \frac{\mathrm{Nu}_{x}}{\sqrt{\mathrm{Re}_{x}}}=-\theta^{\prime}(0),
$$

where $\operatorname{Re}_{x}=U x / \nu$ is the local Reynolds number.

\section{Solution Using the Successive Relaxation Method}

The Successive Relaxation Method has been used with great success to solve boundary problems; see, for example, [2729]. For the current problem, the method begins by letting $p=f^{\prime}$ so that $p^{\prime}=f^{\prime \prime}, p^{\prime \prime}=f^{\prime \prime \prime}$, and (6) becomes

$$
(1+2 \gamma \eta) p^{\prime \prime}+2 \gamma p^{\prime}+f p^{\prime}-p^{2}-K p=0,
$$

but (7) remains unchanged. Proceeding in a manner similar to the Gauss-Seidel method, we replace both (6) and (12) with the iterative scheme defined by

$$
\begin{gathered}
f_{r+1}^{\prime}=p_{r} \\
(1+2 \gamma \eta) p_{r+1}^{\prime \prime}+2 \gamma p_{r+1}^{\prime}+f_{r+1} p_{r+1}^{\prime}-p_{r}^{2}-K p_{r+1}=0, \\
(1+2 \gamma \eta) \theta_{r+1}^{\prime \prime}+2 \gamma \theta_{r+1}^{\prime}+\operatorname{Pr} f \theta_{r+1}^{\prime} \\
+\epsilon\left[(1+2 \gamma \eta)\left(\theta_{r}^{\prime 2}+\theta_{r} \theta_{r}^{\prime \prime}\right)+2 \gamma \theta_{r} \theta_{r}^{\prime}\right]=0,
\end{gathered}
$$

where it follows from boundary conditions (8) and (9) that

$$
\begin{array}{cl}
f_{r+1}(0)=0, & \\
p_{r+1}(0)=1, & p_{r+1}(\infty)=0, \\
\theta_{r+1}(0)=1, & \theta_{r+1}(\infty)=0 .
\end{array}
$$

Equations (13)-(15) subject to conditions (16)-(18) will be solved using the Chebyshev Spectral Collocation method. Before we do this, we start by using the domain truncation method to replace the semi-infinite interval $[0, \infty)$ with the finite interval $[0, L]$ on the $\eta$ axis, where $L$ is sufficiently large. For the sake of convenience, we use the transformation

$$
\eta(\xi)=\frac{L}{2}(\xi+1) ; \quad-1 \leq \xi \leq 1
$$

to map interval $[0, L]$ on the $\eta$ axis to interval $[-1,1]$ on the $\xi$ axis. On the latter, we form a computational grid using the Chebyshev collocation points generated by the formula

$$
\eta_{j}=\cos \left(\frac{\pi p}{N}\right) ; \quad j=0,1, \ldots, N .
$$

When Chebyshev differentiation [30] is applied on iterative scheme defined by (13)-(18), we end up with the discrete form

$$
\begin{gathered}
A_{1} f_{r+1}=B_{1}, \quad f_{r+1}\left(\xi_{N}\right)=0, \\
A_{2} p_{r+1}=B_{2}, \quad p_{r+1}\left(\xi_{N}\right)=1, \quad p_{r+1}\left(\xi_{0}\right)=0, \\
A_{3} \Theta_{r+1}=B_{3}, \quad \theta_{r+1}\left(\xi_{N}\right)=1, \quad \theta_{r+1}\left(\xi_{0}\right)=0,
\end{gathered}
$$

where

$$
\begin{gathered}
A_{1}=D, \quad B_{1}=p_{r}, \\
A_{2}=(1+2 \gamma \eta) D^{2}+\operatorname{diag}\left(2 \gamma+f_{r+1}\right) D-K I, \quad B_{2}=\underset{r}{p_{r}^{2}} \\
A_{3}=(1+2 \gamma \eta) D^{2}+\operatorname{diag}\left(2 \gamma+\operatorname{Pr} f_{r+1}\right) D \\
B_{3}=\epsilon\left[(1+2 \gamma \eta)\left(\Theta_{r}^{\prime 2}+\Theta_{r} \Theta_{r}^{\prime \prime}\right)+2 \gamma \Theta \Theta^{\prime}\right]
\end{gathered}
$$

As a consequence of the transformation (19), we have $D=$ (2/L) $\widehat{D}$ where $\widehat{D}$ is the Chebyshev differentiation matrix. Since (21)-(23) are decoupled, they may be solved seperately. This is preceded by applying boundary conditions as illustrated below:

$$
\begin{aligned}
& \left(\begin{array}{cccc} 
& & & \\
& A_{1} & \\
\hline 0 & \cdots & 0 & 1
\end{array}\right)\left(\begin{array}{c}
f_{r+1}\left(\xi_{0}\right) \\
\vdots \\
\hline f_{r+1}\left(\xi_{N}\right)
\end{array}\right)=\left(\begin{array}{c}
B_{2} \\
\hline 0
\end{array}\right), \\
& \left(\begin{array}{ccccc}
1 & 0 & & \cdots & 0 \\
\hline & & A_{3} & & \\
\hline 0 & \cdots & & 0 & 1
\end{array}\right)\left(\begin{array}{c}
\frac{\theta_{r+1}\left(\xi_{0}\right)}{\vdots} \\
\frac{\vdots}{\theta_{r+1}\left(\xi_{N}\right)}
\end{array}\right)=\left(\begin{array}{c}
0 \\
\frac{B_{2}}{1}
\end{array}\right) \text {, } \\
& \left(\begin{array}{ccccc}
1 & 0 & & \cdots & 0 \\
\hline & & A_{2} & & \\
\hline 0 & \cdots & & 0 & 1
\end{array}\right)\left(\begin{array}{c}
\frac{p_{r+1}\left(\xi_{0}\right)}{\vdots} \\
\frac{p_{r+1}\left(\xi_{N}\right)}{p^{2}}
\end{array}\right)=\left(\begin{array}{c}
0 \\
\frac{B_{1}}{1}
\end{array}\right) \text {. }
\end{aligned}
$$

In order to satisfy the boundary conditions (16), (17), and (18), we choose initial approximations

$$
\begin{gathered}
f_{0}(\eta)=1-e^{-\eta}, \\
p_{0}(\eta)=\theta_{0}(\eta)=e^{-\eta},
\end{gathered}
$$

which if used with iterative scheme defined by (13)-(18) generates subsequent approximations $f_{r}, p_{r}, \theta_{r}$ for each $r=$ $1,2,3, \ldots$.

\section{Results and Discussion}

In this section, we give the SRM results for the main parameters that have significant effects on the fluid flow velocity and temperature. We remark that all the SRM results presented in this work were obtained using $N=50$ collocation points, and also convergence was achieved after as few as five iterations. Also the infinity value $\left(\eta_{\infty}\right)$ was taken to be 50 . Unless otherwise stated, the default values for the parameters are taken as $\operatorname{Pr}=0.71 ; \gamma=0.1 ; \epsilon=0.2 ; K=1$. In order to validate the numerical method, it was compared with the MATLAB routine $b v p 4 c$ which is an adaptive Lobatto quadrature iterative scheme. Table 1 presents a comparison between SRM approximate results, the $b v p 4 c$ results and previous results for $\operatorname{Pr}=1$, and all other parameters being equal to zero. From this table, we clearly observe that the SRM results excellently agree with the $b v p 4 c$ results up to eight significant figures; whereas previously obtained results are 
TABLE 1: Comparison of the SRM results of $-\theta^{\prime}(0)$ with those obtained by $b v p 4 c$ as well as previously obtained results.

\begin{tabular}{ccccccc}
\hline $\operatorname{Pr}$ & & & $-\theta^{\prime}(0)$ & & & \\
& {$[14]$} & {$[15]$} & {$[16]$} & SRM & bvp4c \\
\hline 1.0 & 0.5820 & 0.5820 & 0.5801 & 0.5821 & 0.58197671 & 0.58197671 \\
\hline
\end{tabular}

TABLE 2: Comparison of the SRM results of $-f^{\prime \prime}(0),-\theta^{\prime}(0)$ with those obtained by $b v p 4 c$ for different values of the curvature parameter.

\begin{tabular}{lcccc}
\hline \multirow{2}{*}{$-f^{\prime \prime}(0)$} & \multicolumn{2}{c}{$-\theta^{\prime}(0)$} \\
& $b v p 4 c$ & SRM & $b v p 4 c$ & SRM \\
\hline 0.0 & 1.41421356 & 1.41421356 & 0.33010615 & 0.33010615 \\
0.25 & 1.52316810 & 1.52316810 & 0.37955209 & 0.37955209 \\
0.5 & 1.62648794 & 1.62648794 & 0.42630480 & 0.42630480 \\
\hline
\end{tabular}

correct only to four significant figures. It can also be remarked that the cpu time for SRM is significantly less than that of bvp $4 c$ method. Convergence is achieved by SRM with as few as ten iterations.

Table 2 displays the effect of the curvature parameter on the skin friction and Nusselt number when all other parameters are kept constant. Both the temperature and velocity gradients at the surface are larger for larger values of curvature parameter $(\gamma)$, which produces larger skin friction coefficient and Nusselt number. The effect of the permeability parameter $K$ and thermal conductivity parameter $\epsilon$, on the skin friction and Nusselt number, are, respectively, depicted on Table 3. We observe clearly from this table that the absolute values of $f^{\prime \prime}(0)$ are increased by increasing the permeability parameter. Hence, in order to minimize the skin friction value which is usually looked for in an industrial application, one needs to reduce the permeability of the medium. The Nusselt number is reduced as the thermal conductivity of the material increases.

The effects of the pertinent physical parameters in this study on the velocity components and temperature distributions are depicted from Figures 1 to 7 . Figure 1 displays the effect of the curvature parameter $\gamma$ on the horizontal velocity profiles. We clearly observe in this figure that the effect of this parameter on the horizontal component is very insignificant within the dynamic region [0,0.7] near the surface. Outside this region, we observe that this velocity component increases as $\gamma$ increases.

The influence of the permeability parameter on the horizontal velocity is depicted in Figure 2. From this figure, we observe that when the permeability parameter is increased, the velocity boundary layer is decreased. Thus, the fluid velocity in the horizontal direction decreases. Figure 3 shows the effect of the curvature of the cylinder on the transverse velocity profiles. We observe that the transverse velocity component is insignificantly affected by curvature parameter within the dynamic region $[0,1.3]$. Outside this dynamic region, we see that as the curvature of the stretching cylinder increases, this velocity component increases. Physically, as $\gamma \rightarrow 0$, the outer surface of the cylinder behaves like a flat surface. Thus, as $\gamma$ increases, the viscosity effect is reduced

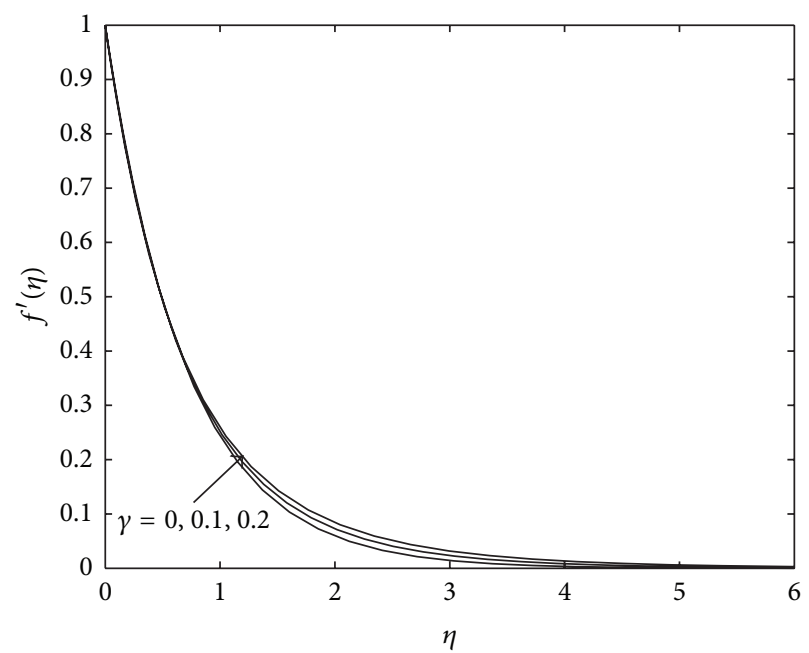

FIGURE 1: Graph of the SRM solutions for the horizontal velocity for different values of $\gamma$.

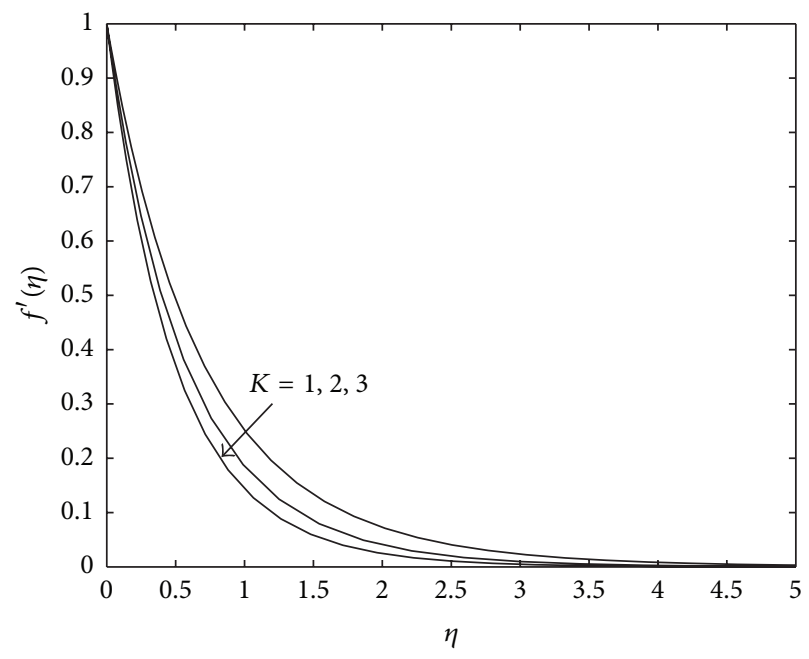

FIgURE 2: Effect of the $K$ on the horizontal velocity profiles.

due to that the contact area of the surface with fluid tends to the tangential position.

In Figure 4, we display the effect of the permeability parameter $K$ on the transverse velocity profiles. The transverse velocity profiles are greatly reduced as the permeability parameter increases. Figure 5 shows the effect of the curvature parameter on the temperature profiles. It can be clearly observed in this figure that there is no significant effect within the dynamic region less than a unit. However, after this region the temperature is enhanced by increasing the values of the curvature parameter. Figure 6 displays 
TABLE 3: Comparison of the SRM results of $-f^{\prime \prime}(0),-\theta^{\prime}(0)$ with those obtained by $b v p 4 c$ for different values of the permeability parameter and thermal conductivity parameter, respectively.

\begin{tabular}{|c|c|c|c|c|c|}
\hline \multirow{2}{*}{$K$} & \multicolumn{2}{|c|}{$-f^{\prime \prime}(0)$} & \multirow{2}{*}{$\epsilon$} & \multicolumn{2}{|c|}{$-\theta^{\prime}(0)$} \\
\hline & bvp $4 c$ & SRM & & bvp $4 c$ & SRM \\
\hline 1.0 & 1.50188161 & 1.50188161 & 0.0 & 0.43791391 & 0.4379139 \\
\hline 2.0 & 1.82359290 & 1.82359290 & 0.2 & 0.37955209 & 0.37955209 \\
\hline 3.0 & 2.09341552 & 2.09341552 & 0.4 & 0.32962783 & 0.32962783 \\
\hline
\end{tabular}

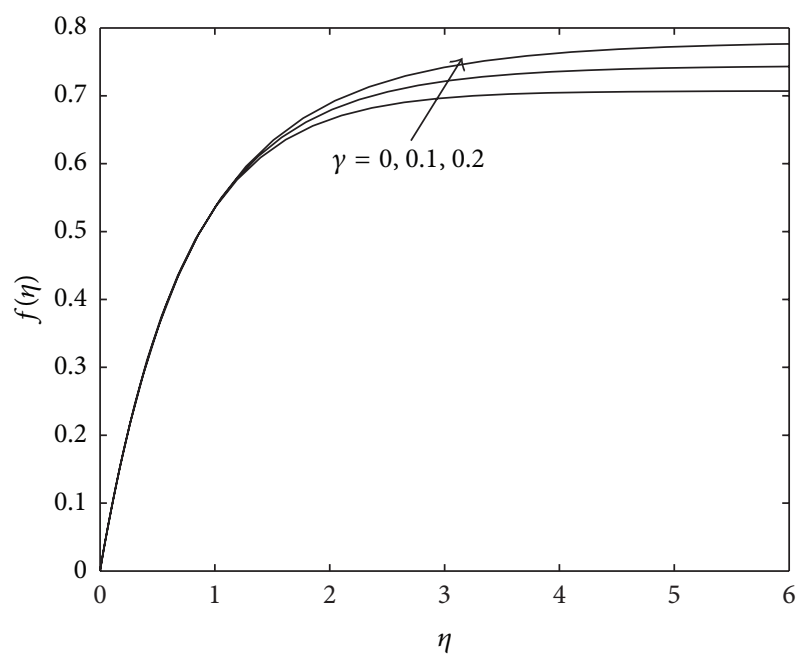

FIGURE 3: Graph of the SRM solutions of the transverse velocity for different values of $\gamma$.

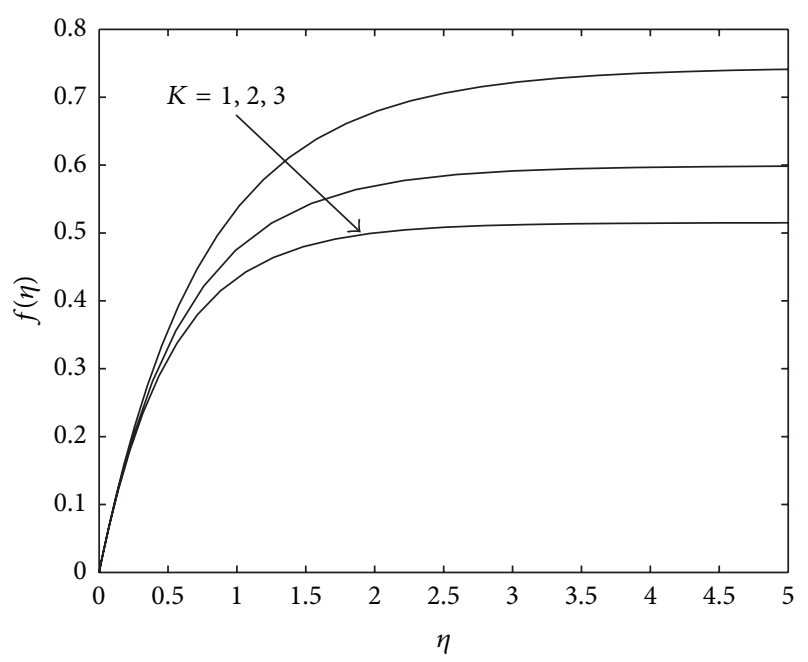

FIGURE 4: Effect of the $K$ on the $v$-velocity profiles.

the effect of varying thermal conductivity on the temperature distribution. As expected, increasing thermal conductivity (or a higher viscosity) results in the thinning of the thermal boundary layer and hence higher heat transfer rate at the surface. This is clearly depicted in Figure 7.

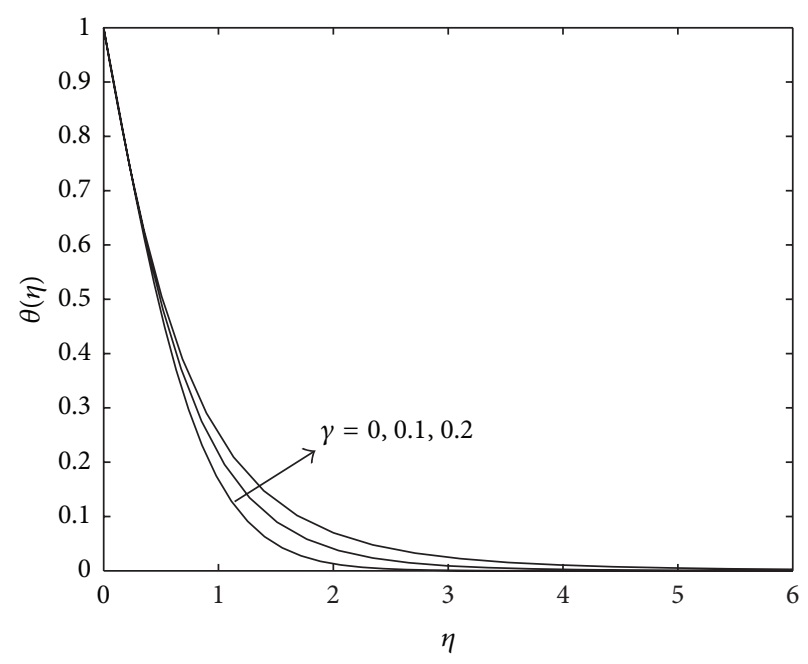

FIGURE 5: Graph of the SRM solutions of the temperature profiles for different values of $\gamma$.

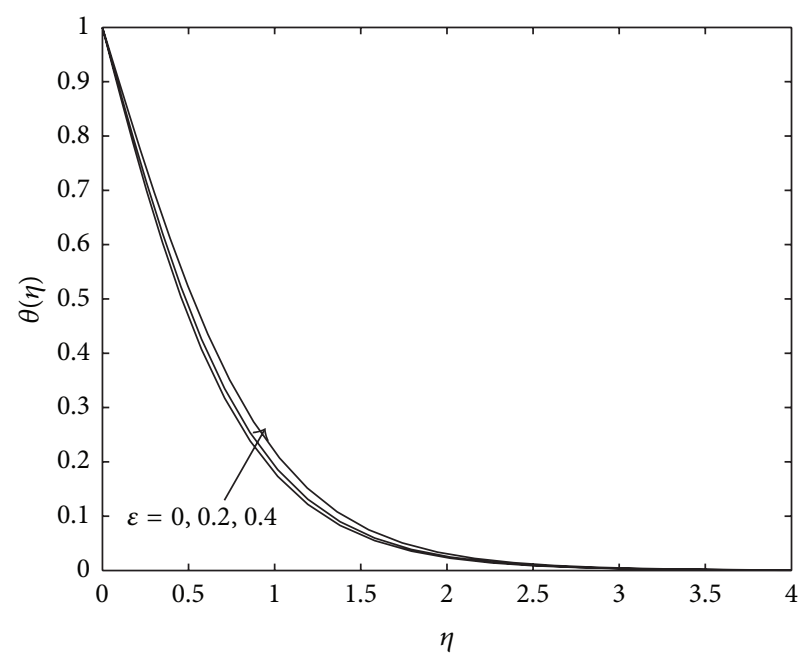

Figure 6: Effect of the $\epsilon$ on the temperature profiles.

\section{Conclusion}

We have numerically analyzed the problem of laminar boundary layer flow and heat transfer along a stretching cylinder embedded in a porous medium with variable conductivity applying a recently developed numerical method. The system of partial differential equations governing 


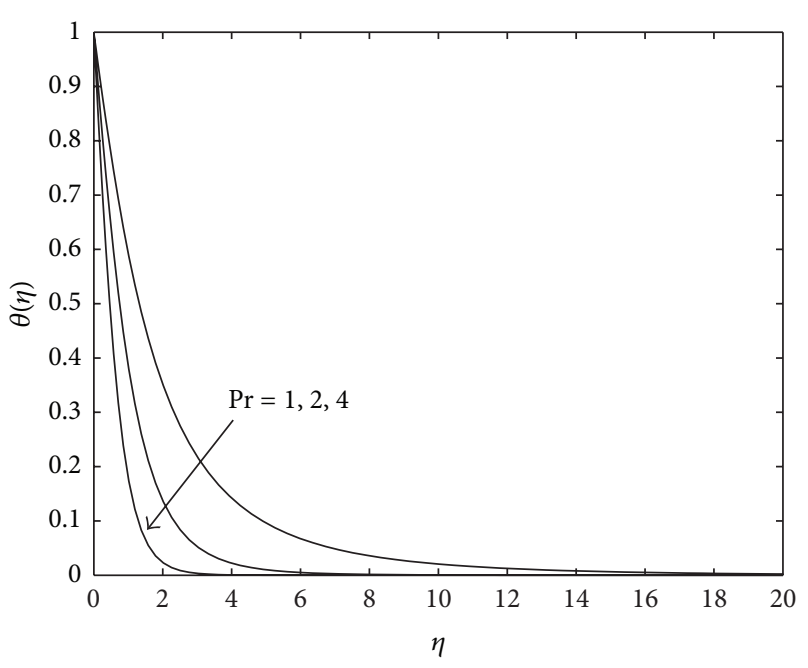

FIgUre 7: Effect of the Pr on the temperature profiles.

the current problem was transformed into a set of ordinary differential equations by using appropriate similarity transformations. The investigation observed that the curvature of the stretching cylinder has a very significant effect on both the velocity and temperature fields. Both the skin friction coefficient and local Nusselt number increase as the curvature increases. The flow properties were found to be significantly influenced by the permeability parameter. The present study also shows that the SRM is a very reliable, easy, and accurate method which we trust that it can be used to solve even more complex and complicated systems. It is hoped that the current findings can be used as the basis for many scientific and engineering applications.

\section{Conflict of Interests}

The authors declare that there is no conflict of interests in the use of the aforementioned software.

\section{Acknowledgment}

The authors wish to acknowledge financial support from the University of Venda and NRF.

\section{References}

[1] B. C. Sakiadis, "Boundary-layer behaviour on continuous solid surfaces: I. Boundary-layer equations for two-dimensional and axisymmetric flow," AIChE Journal, vol. 7, pp. 26-28, 1961.

[2] B. C. Sakiadis, "Boundary-layer behaviour on continuous solid surfaces: II. Boundary-layer equations for two-dimensional and axisymmetric flow," AIChE Journal, vol. 7, pp. 221-225, 1961.

[3] L. J. Crane, "Flow past a stretching plate," Zeitschrift für angewandte Mathematik und Physik, vol. 12, pp. 645-647, 1970.

[4] M. K. Laha, P. S. Gupta, and A. S. Gupta, "Heat transfer characteristics of the flow of an incompressible viscous fluid over a stretching sheet,"Wärme- und Stoffübertragung, vol. 24, no. 3, pp. 151-153, 1989.
[5] N. Afzal, "Heat transfer from a stretching surface," International Journal of Heat and Mass Transfer, vol. 36, no. 4, pp. 1128-1131, 1993.

[6] K. V. Prasad, S. Abel, and P. S. Datti, "Diffusion of chemically reactive species of a non-Newtonian fluid immersed in a porous medium over a stretching sheet," International Journal of NonLinear Mechanics, vol. 38, no. 5, pp. 651-657, 2003.

[7] M. S. Abel and N. Mahesha, "Heat transfer in MHD viscoelastic fluid flow over a stretching sheet with variable thermal conductivity, non-uniform heat source and radiation," Applied Mathematical Modelling, vol. 32, no. 10, pp. 1965-1983, 2008.

[8] M. S. Abel, P. G. Siddheshwar, and M. M. Nandeppanavar, "Heat transfer in a viscoelastic boundary layer flow over a stretching sheet with viscous dissipation and non-uniform heat source," International Journal of Heat and Mass Transfer, vol. 50, no. 5-6, pp. 960-966, 2007.

[9] R. C. Bataller, "Viscoelastic fluid flow and heat transfer over a stretching sheet under the effects of a non-uniform heat source, viscous dissipation and thermal radiation," International Journal of Heat and Mass Transfer, vol. 50, no. 15-16, pp. 31523162, 2007.

[10] E. M. E. Elbarbary and N. S. Elgazery, "Flow and heat transfer of a micropolar fluid in an axisymmetric stagnation flow on a cylinder with variable properties and suction (numerical study)," Acta Mechanica, vol. 176, no. 3-4, pp. 213-229, 2005.

[11] P. Datta, D. Anilkumar, S. Roy, and N. C. Mahanti, "Effect of non-uniform slot injection (suction) on a forced flow over a slender cylinder," International Journal of Heat and Mass Transfer, vol. 49, no. 13-14, pp. 2366-2371, 2006.

[12] H. T. Lin and Y. P. Shih, "Buoyancy effects on the laminar boundary layer heat transfer along vertically moving cylinders," Journal of the Chinese Institute of Engineers, vol. 4, no. 1, pp. 4751, 1981.

[13] H. T. Lin and Y. P. Shih, "Laminar boundary layer heat transfer along static and moving cylinders," Journal of the Chinese Institute of Engineers, vol. 3, no. 1, pp. 73-79, 1980.

[14] A. Ishak and R. Nazar, "Laminar boundary layer flow along a stretching cylinder," European Journal of Scientific Research, vol. 36, no. 1, pp. 22-29, 2009.

[15] L. J. Grubka and K. M. Bobba, "Heat transfer characteristics of a continuous stretching surface with variable temperature," Journal of Heat Transfer, vol. 107, no. 1, pp. 248-250, 1985.

[16] M. E. Ali, "Heat transfer characteristics of a continuous stretching surface," Heat and Mass Transfer, vol. 29, no. 4, pp. 227-234, 1994.

[17] S. Mukhopadhyay, "Mixed convection boundary layer flow long a stretching cylinder in medium," Journal of Petroleum Science and Engineering, vol. 96-97, pp. 73-78, 2012.

[18] S. Mukhopadhyay, "Analysis of boundary layer flow and heat transfer along a stretching cylinder in a porousMedium," ISRN Thermodynamics, vol. 2012, Article ID 704984, 7 pages, 2012.

[19] R. R. Rangi and N. Ahmad, "Boundary layer flow past a stretching cylinder and heat transfer with variable thermal conductivity," Applied Mathematics, vol. 3, no. 3, pp. 205-209, 2012.

[20] E. M. A. Elbashbeshy, T. G. Emam, M. S. El-Azab, and K. M. Abdelgaber, "Laminar boundary layer flow along a stretching cylinder embedded in a porous medium," International Journal of the Physical Sciences, vol. 7, no. 24, pp. 3067-3072, 2012.

[21] K. Vajravelu, K. V. Prasad, and C.-O. Ng, "Unsteady convective boundary layer flow of a viscous fluid at a vertical surface 
with variable fluid properties," Nonlinear Analysis: Real World Applications, vol. 14, no. 1, pp. 455-464, 2013.

[22] S. Sarkar, S. Ganguly, and A. Dalal, "Buoyancy driven flow and heat transfer of nanofluids past a square cylinder in vertically upward flow," International Journal of Heat and Mass Transfer, vol. 59, pp. 433-450, 2013.

[23] S. K. Soid, K. M. Yusof, A. S. Abd Aziz, and S. A. Kechil, "Magnetohydrodynamics boundary layer flows over a stretching surface with radiation effect and embedded in porous medium," World Academy of Science, Engineering and Technology, vol. 68, pp. 1769-1773, 2012.

[24] E. M. A. Elbashbeshy, T. G. Emam, M. S. El-Azab, and K. M. Abdelgaber, "Laminar boundary layer flow along a stretching horizontal cylinder embedded in a porous medium in the presence of a heat source or sink with suction/injection," International Journal of Energy \& Technology, vol. 4, no. 28, pp. $1-6,2012$.

[25] D. Chatterjee and M. Raja, "Mixed convection heat transfer past in-line square cylinders in a vertical duct," Thermal Science, vol. 17, no. 2, pp. 567-580, 2013.

[26] S. Mukhopadhyay and R. S. R. Gorla, "Slip effects on boundary layer flow and heat transfer along a stretching cylinder," International Journal of Applied Mechanics and Engineering, vol. 18, no. 2, pp. 447-459, 2013.

[27] S. S. Motsa, P. Dlamini, and M. Khumalo, "A new multistage spectral relaxation method for solving chaotic initial value systems," Nonlinear Dynamics, vol. 72, no. 1-2, pp. 265-283, 2013.

[28] S. Shateyi and O. D. Makinde, "Hydromagnetic stagnationpoint flow towards a radially stretching convectively heated disk," Mathematical Problems in Engineering, vol. 2013, Article ID 616947, 8 pages, 2013.

[29] S. S. Motsa, O. D. Makinde, and S. Shateyi, "On the successive linearisation approach to the flow of reactive third-grade liquid in a channel with isothermal walls," Mathematical Problems in Engineering, vol. 2013, Article ID 635392, 7 pages, 2013.

[30] L. N. Trefethen, Spectral Methods in MATLAB, vol. 10 of Software, Environments, and Tools, SIAM, Philadelphia, Pa, USA, 2000. 


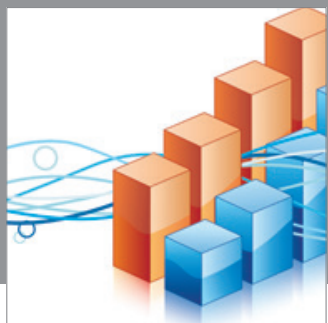

Advances in

Operations Research

mansans

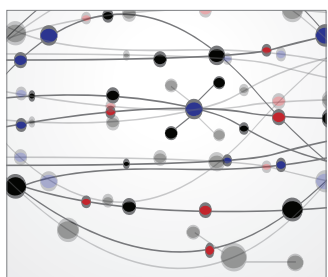

The Scientific World Journal
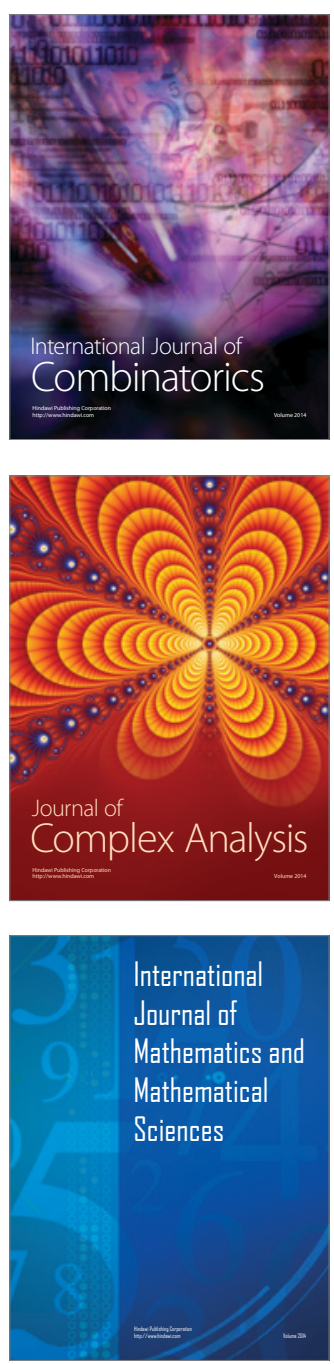
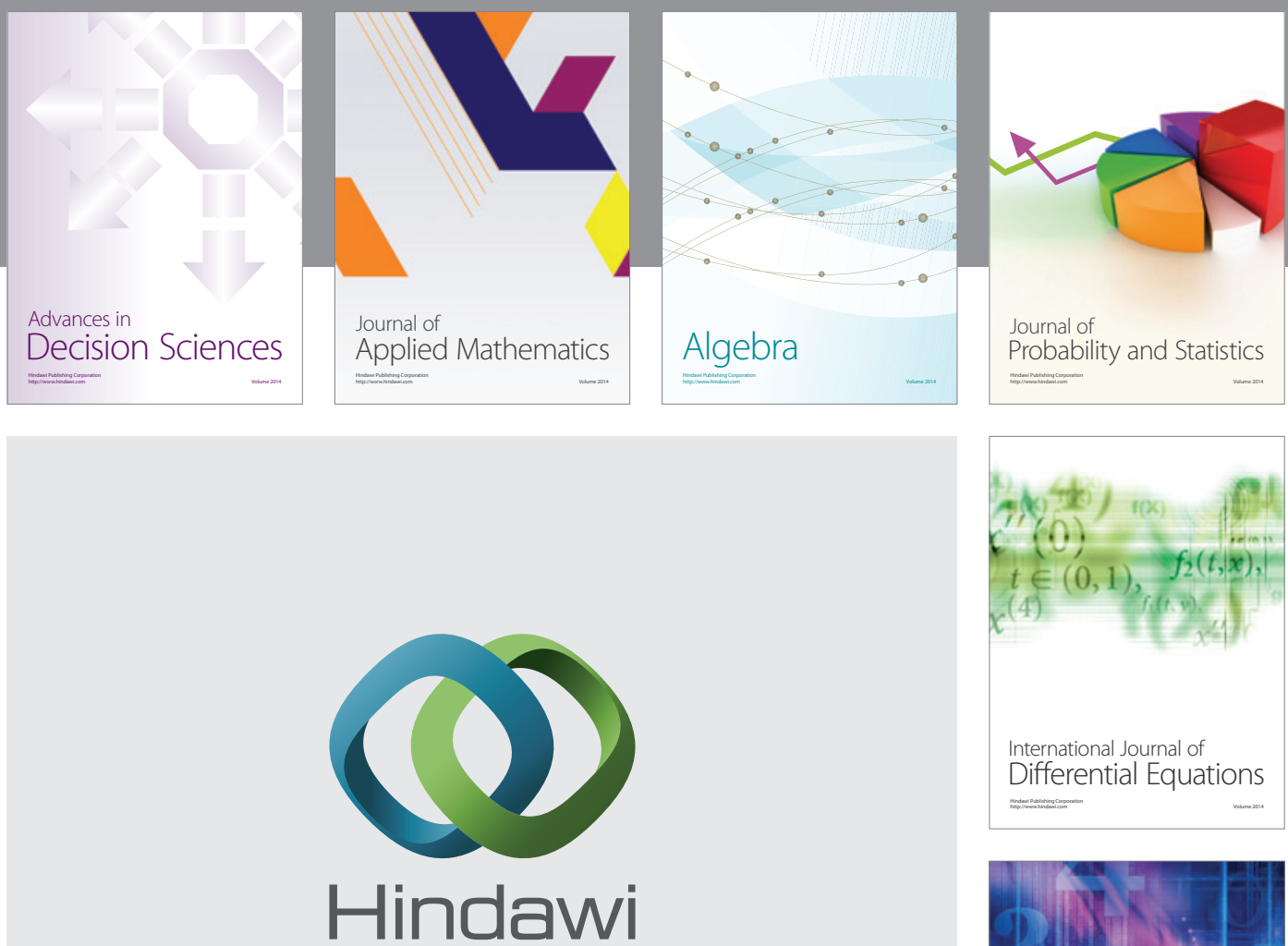

Submit your manuscripts at http://www.hindawi.com
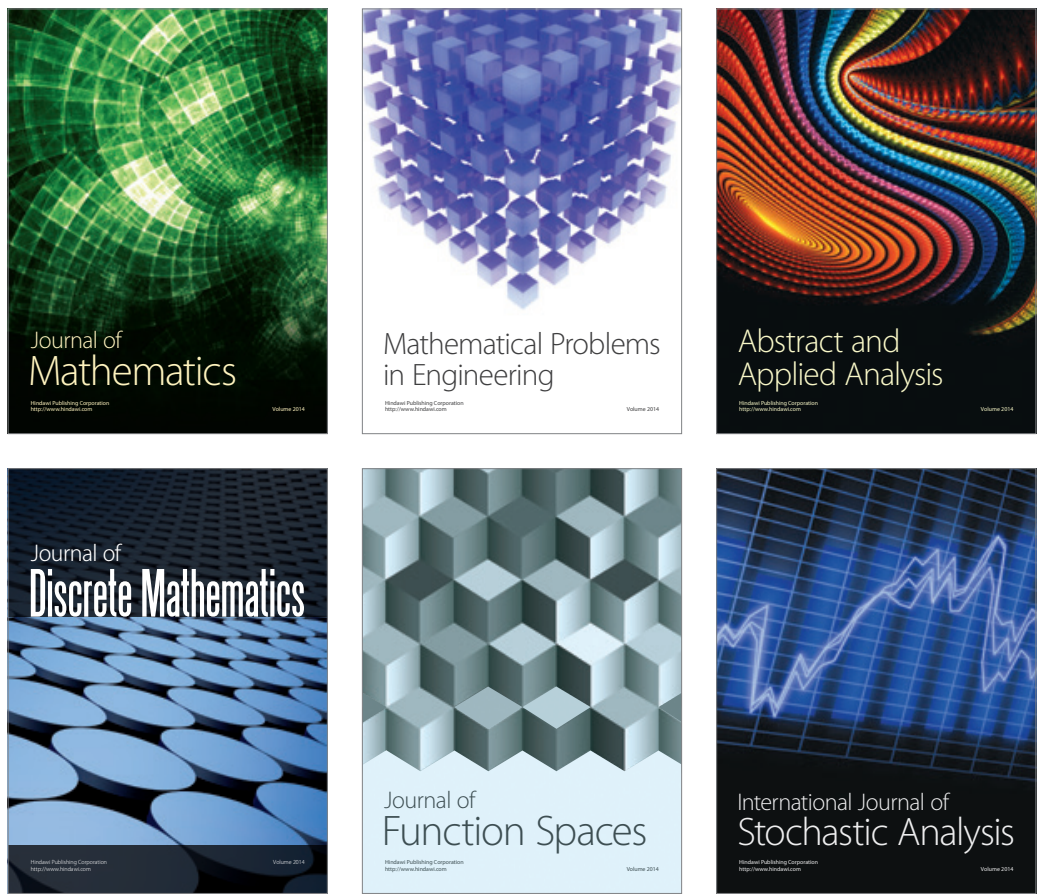

Journal of

Function Spaces

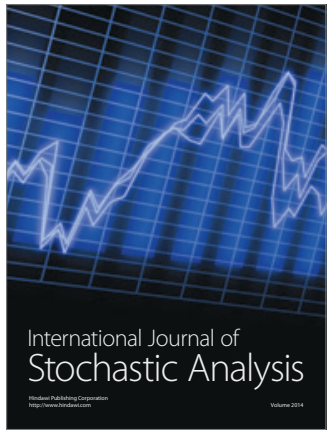

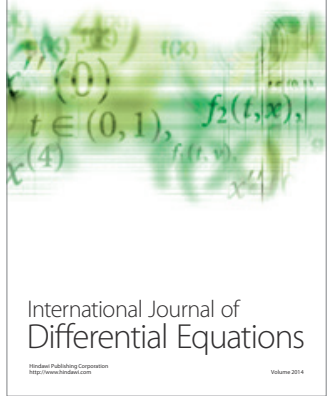
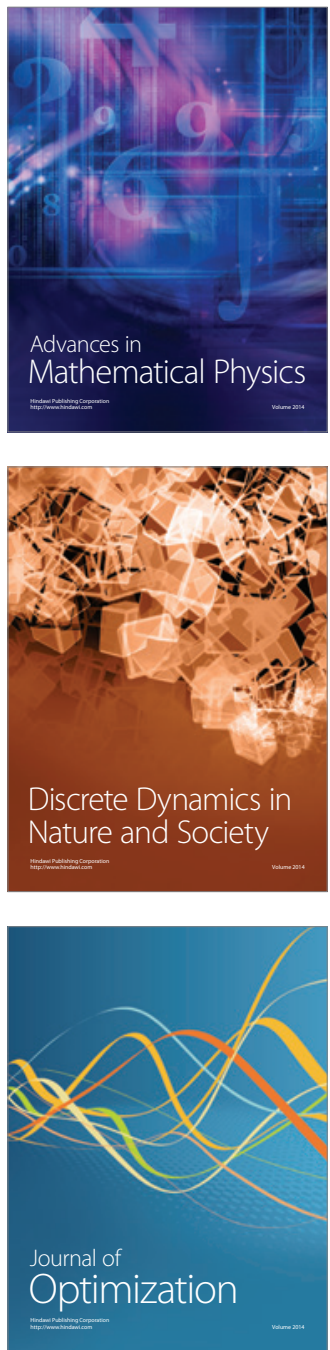